\title{
COVID-19's impact on internet gaming disorder among children and adolescents
}

\author{
Rabica Shahid, MD, Suneeta Kumari, MD, and Stacy Doumas, MD
}

T he impact of the COVID-19 pandemic on the well-being of youth has been significant. Its possible effects range from boredom, depression, anxiety, and suicidal ideation to potential increased rates of internet gaming disorder (IGD), which may have worsened during a nationwide shutdown and extended period of limited social interactions. Presently, there is a paucity of research on the impact of internet gaming on children and adolescents' mental health and well-being during COVID-19. This article aims to bring awareness to the possible rising impact of the COVID-19 pandemic on IGD and mental health in youth.

\section{Gaming offers benefits_-and risks}

The gaming industry has grown immensely over the past several years. While many businesses were impacted negatively during the pandemic, the gaming industry grew. It was estimated to be worth $\$ 159.3$ billion in 2020, an increase of $9.3 \%$ from $2019 .{ }^{1}$

Stay-at-home orders and quarantine protocols during the COVID-19 pandemic have significantly disrupted normal activities, resulting in increased time for digital entertainment, including online gaming and related activities. Internet gaming offers some benefits for children and adolescents, including socialization and connection with peers, which was especially important for avoiding isolation during the pandemic. Empirical evidence of the positive effects of internet gaming can be seen in studies of youth undergoing chemotherapy, those receiving psychotherapy for anxiety or depression, and those having emotional and behavioral problems. ${ }^{2}$ Internet gaming also provides participants with a platform to communicate with the outside world while maintaining social distancing, and might reduce anxiety, and in some cases, depression. ${ }^{3}$

Despite these benefits, for some youth, excessive internet gaming can have adverse effects. Due to its addictive properties, internet gaming can be dangerous for vulnerable individuals and lead to unhealthy habits, such as disturbed sleep patterns and increased anxiety. ${ }^{4}$ In a cross-sectional study conducted in China, Yu et $\mathrm{al}^{5}$ examined the association between IGD and suicidal ideation. They concluded that IGD was positively associated with insomnia and then depression, which in turn contributed to suicide ideation. ${ }^{5}$ A study based on a survey conducted in Iran from May to August 2020 in individuals age 13 to 18 years found that depression, anxiety, and stress were significant mediators in the association between IGD and self-reported quality of life. ${ }^{2}$

Internet gaming disorder is included in DSM-5 as a "condition for further study" and in ICD-11. ${ }^{6}$ Before the COVID-19 pandemic, a study of 1,178 American youth age 8 to 18 years revealed that $8.5 \%$ of gamers met the criteria for IGD. ${ }^{7}$ In a meta-analysis that included 16 studies, the pooled prevalence of IGD among adolescents was $4.6 \%{ }^{8}$ Some countries, including China and South Korea, have developed treatment plans for IGD, ${ }^{6}$ but in the United States treatment guidelines have not been established due to insufficient evidence. ${ }^{9}$

The COVID-19 pandemic has likely led to an increased number of children and
Dr. Shahid is a Research Volunteer, Department of Psychiatry, Jersey Shore University Medical Center, Neptune, New Jersey. Dr. Kumari is a PGY-2 Psychiatry Resident, Department of Psychiatry, Ocean Medical Center, Brick Township, New Jersey. Dr. Doumas is Chief, Child and Adolescent Psychiatry, Residency Program Director, Vice Chair of Education and Research, Department of Psychiatry, Jersey Shore University Medical Center, Neptune, New Jersey, and Vice Chair, Department of Psychiatry, Hackensack Meridian School of Medicine, Nutley, New Jersey.

Disclosures

The authors report no financial relationships with any companies whose products are mentioned in this article, or with manufacturers of competing products.

doi: 10.12788/cp.0152

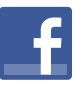

Discuss this article at www.facebook.com/ MDedgePsychiatry 


\section{Clinical Point}

In the United States,

treatment guidelines

for IGD have not

been established

due to insufficient

evidence adolescents with IGD and its adverse effects on their mental health and well-being. It remains to be seen whether these youth will improve as the pandemic resolves and they resume normal activities, or if impairments will persist.

In conclusion, while internet gaming during the COVID-19 pandemic has provided benefits for many children and adolescents, the negative impact for those who develop IGD may be significant. We should be prepared to detect and address the needs of these youth and their families. Additional research is needed on the post-pandemic prevalence of IGD, its impact on youth mental health, and treatment strategies.

\section{References}

1. WePC. Video game industry statistics, trends and data in 2021. Accessed June 7, 2021. https://www.wepc.com/ news/video-game-statistics/
2. Fazeli S, Mohammadi Zeidi I, Lin CY, et al. Depression, anxiety, and stress mediate the associations between internet gaming disorder, insomnia, and quality of life during the COVID-19 outbreak. Addict Behav Rep. 2020;12:100307. doi: 10.1016/j.abrep.2020.100307

3. Özçetin M, Gümüştaş F, Çă̆ Y, et al. The relationships between video game experience and cognitive abilities in adolescents. Neuropsychiatr Dis Treat. 2019;15:1171-1180. doi: 10.2147/NDT.S206271

4. Männikkö N, Ruotsalainen H, Miettunen J, et al. Problematic gaming behaviour and health-related outcomes: a systematic review and meta-analysis. J Health Psychol. 2020;25(1):67-81. doi: 10.1177/1359105317740414

5. Yu $Y$, Yang $X$, Wang $S$, et al. Serial multiple mediation of the association between internet gaming disorder and suicidal ideation by insomnia and depression in adolescents in Shanghai, China. BMC Psychiatry. 2020;20(1):460. doi: 10.1186/s12888-020-02870-zz

6. American Psychiatric Association. Internet gaming. Published June 2018. Accessed June 7, 2021. www. psychiatry.org/patients-families/internet-gaming

7. Gentile D. Pathological video-game use among youth ages 8 to 18: a national study. Psychol Sci. 2009;20(5):594-602. doi: 10.1111/j.1467-9280.2009.02340.x

8. Fam JY. Prevalence of internet gaming disorder in adolescents: A meta-analysis across three decades. Scand J Psychol. 2018;59(5):524-531. doi: 10.1111/sjop.12459

9. Gentile DA, Bailey K, Bavelier D, et al. Internet gaming disorder in children and adolescents. Pediatrics. 2017; 140(suppl 2):S81-S85. doi: 10.1542/peds.2016-1758H 